\title{
Sinonasal Undifferentiated Carcinoma: A Case Report and Review of Literature
}

\author{
${ }^{1}$ Samuel Rajan, ${ }^{2}$ Anoop Raj, ${ }^{3}$ Anju Chauhan, ${ }^{4}$ PK Rathore, ${ }^{5}$ Vikram Wadhwa, ${ }^{6}$ Nita Khurana, ${ }^{7}$ Chandala Chitguppi
}

\begin{abstract}
Sinonasal undifferentiated carcinoma (SNUC) is a rare tumor of the nasal cavity and paranasal sinuses. It can be differentiated from other neoplasms by correlating clinical, light microscopic, immunohistochemical and ultrastructural characteristics. Due to the small number of reported cases, the ideal treatment regimen has not been systematically evaluated. The treatment of SNUC includes aggressive multimodality therapy, including surgical resection and adjuvant treatment (i.e. radiotherapy, chemotherapy) because of high rate of both loco-regional recurrence and distant metastasis.
\end{abstract}

Keywords: Carcinoma, Paranasal sinu, Sinonasal, Undifferentiated.

How to cite this article: Rajan S, Raj A, Chauhan A, Rathore PK, Wadhwa V, Khurana N, Chitguppi C. Sinonasal Undifferentiated Carcinoma: A Case Report and Review of Literature. Clin Rhinol An Int J 2015;8(2):60-63.

Source of support: Nil

Conflict of interest: None

\section{INTRODUCTION}

Sinonasal undifferentiated carcinoma (SNUC) is a rare tumor of the nasal cavity and paranasal sinuses first described by Frierson in $1986 .{ }^{1}$ Olfactory neuroblastoma and SNUC may appear quite similar on histological examination. Due to the fact that olfactory neuroblastoma has a much better prognosis, a distinction with SNUC has to be drawn. ${ }^{4}$ Confirming the diagnosis of SNUC at the light microscopic level can be challenging, since the microscopic differential diagnosisincludes olfactory neuroblastoma, rhabdomyosarcoma, undifferentiated nasopharyngeal carcinoma (lymphoepithelioma), malignant lymphoma, malignant melanoma, and neuroendocrine

\footnotetext{
${ }^{1,3}$ Senior Resident, ${ }^{2}$ Director, Professor and Head

${ }^{4}$ Director and Professor, ${ }^{5}$ Specialist, ${ }^{6}$ Professor

${ }^{7}$ Junior Resident

${ }^{1-5,7}$ Department of Otorhinolaryngology, Maulana Azad Medical College, New Delhi, India

${ }^{6}$ Department of Pathology, Maulana Azad Medical College New Delhi, India
}

Corresponding Author: Samuel Rajan, Senior Resident Department of Otorhinolaryngology, Maulana Azad Medical College, New Delhi, India, Phone: 9891071654, e-mail: samuel.aiims@gmail.com carcinoma. Sinonasal undifferentiated carcinoma can be differentiated from these other neoplasms by correlating clinical, light microscopic, immunohistochemical and ultrastructural characteristics. ${ }^{3}$ Aggressive multimodal therapy can provide the best opportunity for local control of this neoplastic process, but the optimal treatment has yet to be determined. The purpose of this report is to present a case of SNUC and to review the current concepts of management for this entity.

\section{CASE REPORT}

A 17 years old male presented to the outpatient department with complaints of epistaxis and nasal obstruction since 2 months, proptosis of right eye since 1 month and progressive reduction in visual acuity over 2 weeks. Examination of nasal cavity revealed a pinkish irregular mass with overlying mucoid discharge filling right nasal cavity which was firm in consistency and bled on probing. Eye examination revealed lateral and outward protrusion of right eye with restriction in range of eye movements and reduced visual acuity. Oral examination was unremarkable. A contrast enhanced computed tomographic scan of the paranasal sinuses and orbits revealed a heterogeneously enhancing soft-tissue mass lesion with its epicenter in bilateral ethmoid sinuses (Fig. 1). This mass

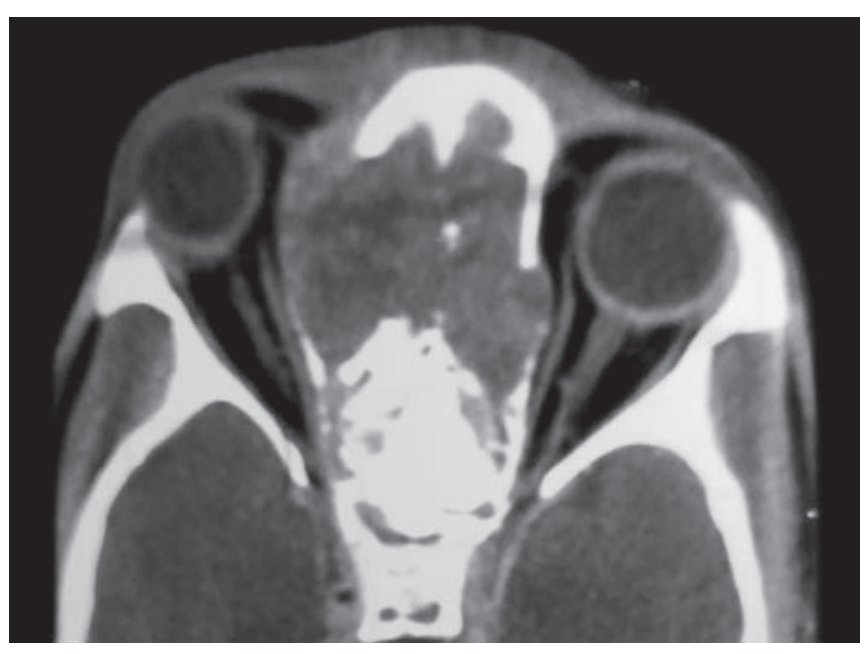

Fig. 1: Contrast-enhanced axial CT scan showing heterogeneously enhancing mass lesion with its epicenter in bilateral ethmoid sinuses and extending posteriorly to involve bilateral sphenoid sinuses. There is destruction of medial bony wall of right orbit with proptosis and lateral displacement of the globe with impingement of the mass on right optic nerve at the apex 
extended inferiorly into bilateral nasal cavities causing destruction of bony septum and right middle turbinate, superolaterally into bilateral frontal sinuses, posteriorly into bilateral sphenoid sinuses and causing destruction of the medial bony wall of right orbit and impinged upon the right optic nerve at the apex (Fig. 2). Biopsy revealed features of undifferentiated carcinoma with large areas of necrosis (Fig. 3). Immunohistochemistry revealed tumor cells which expressed cytokeratin and were negative for vimentin, leukocyte common antigen (LCA), S-100, CD99 and myoglobin (Figs 4A and B). Thus the final diagnosis of SNUC was made. Patient underwent anterior craniofacial resection with right side total maxillectomy and orbital exenteration. The tumor was found to involve the right nasal cavity, bilateral ethmoids and sphenoid sinuses, right maxillary sinus and orbit with optic nerve involvement, and filling both frontal sinuses with destruction of posterior wall and reaching till the

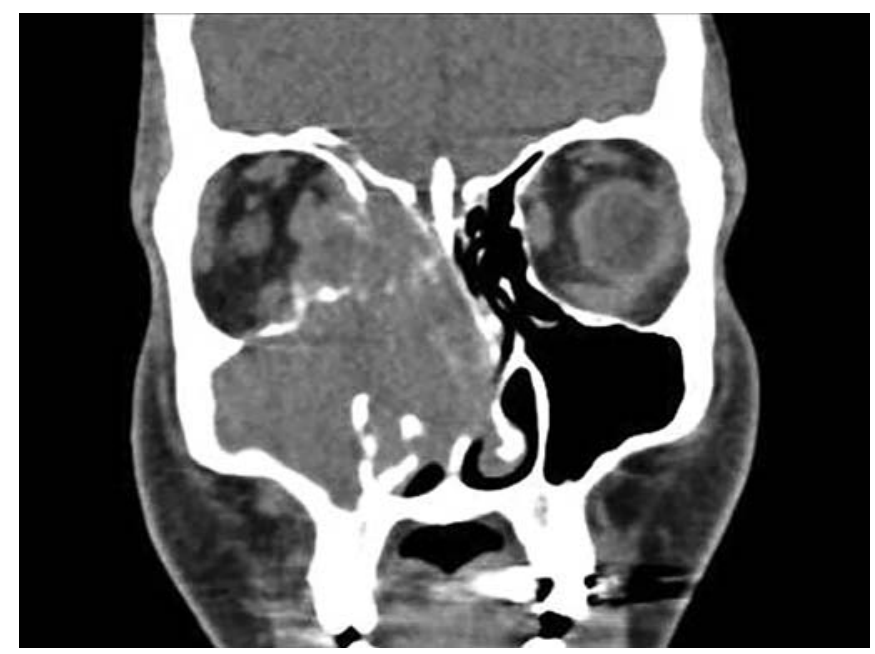

Fig. 2: Contrast-enhanced coronal CT scan showing involvement of right maxillary sinus, anterior ethmoids, the orbit and frontal sinus, with extension into bilateral nasal cavities causing destruction of bony septum and right middle turbinate
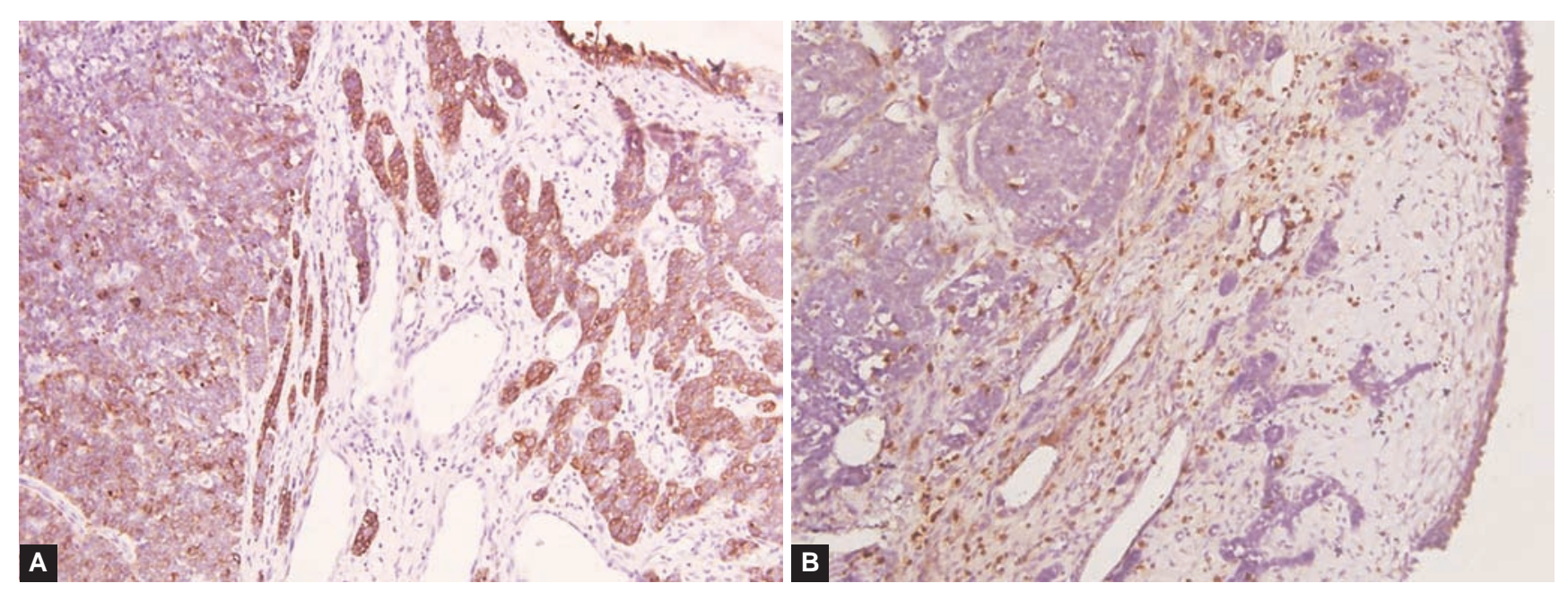

Figs 4A and B: (A) Immunohistochemical staining showing pancytokeratin positive in tumor cells, (B) leukocyte common antigen
negative in tumor cells. Scattered lymphocytes are positive

Figs 4A and B: (A) Immunohistochemical staining showing pancytokeratin positive in tumor cells
negative in tumor cells. Scattered lymphocytes are positive dura but not invading it. The diagnosis was further confirmed on histopathological examination of the surgical specimen and gross resected margins were found to be free of tumor. Postoperative recovery was uneventful with no wound infection, meningitis or cerebrospinal fluid leak. The patient underwent postoperative intensity modulated radiotherapy (IMRT) with a dose of 50 Gy over 6 weeks. The patient is on regular follow-up since last 5 months.

\section{DISCUSSION}

Sinonasal undifferentiated carcinoma was first described in 1986 by Frierson et al as an aggressive neoplasm that was clinicopathologically distinct from other poorly differentiated malignancies of the nasal cavity and sinuses. ${ }^{1}$ It has a slight male predominance (2-3:1) and can affect a broad age range (third to ninth decades), although it most commonly occurs in the sixth decade. ${ }^{2}$ Differential

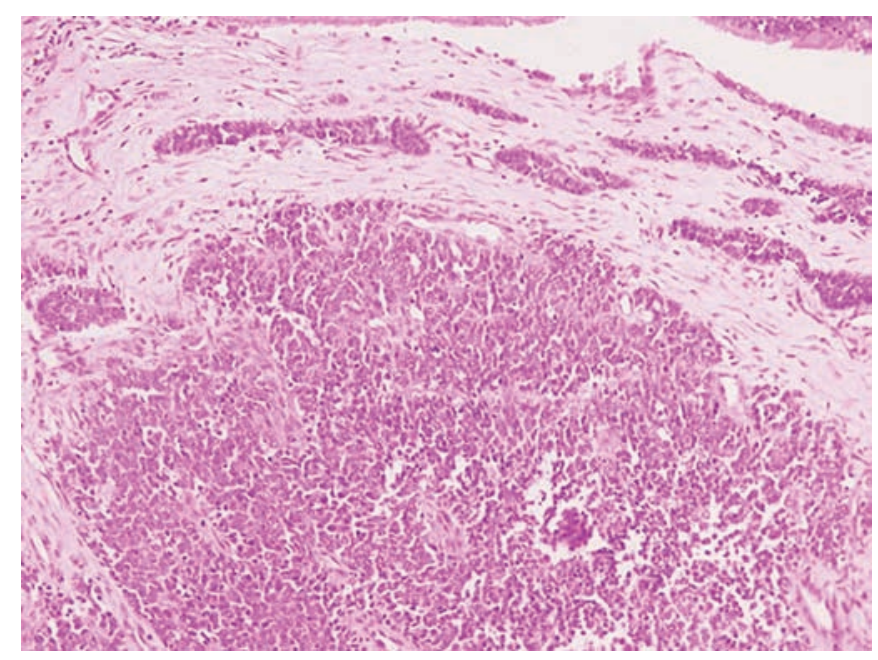

Fig. 3: Photomicrograph of the case showing sheets of large hyperchromatic cells beneath the lining mucosa (Hematoxylin and Eosin stain, magnification 200x) 
diagnoses of such tumors include esthesioneuroblastoma, neuroendocrine carcinoma (SNEC), rhabdomyosarcoma, lymphoepithelioma, lymphoma, melanoma and poorly differentiated adenoid cystic carcinoma. However, this tumor can be distinguished by correlating clinical, light microscopic and immunohistochemical features. ${ }^{3}$

Olfactory neuroblastoma typically presents as a polypoidal mass protruding from the roof of the nasal cavity and consist of lobules of small cells with eosinophilic fibrillary intercellular material in a highly vascular stroma. The tumor cells have indistinct borders with a round nucleus, fine chromatin and little cytoplasm. Nuclear pleomorphism is inconspicuous and mitotic activity is generally low. Homer Wright pseudorosettes are common and ganglion cells may occasionally be seen. Immunohistochemistry demonstrates staining for neuroendocrine markers neuron-specific enolase (NSE), synaptophysin and chromogranin) and S-100 is usually positive in a sustentacular pattern. Ultrastructurally, there is evidence of profuse neurosecretory granules and neurofilaments. ${ }^{4}$

Differential diagnosis of SNUC from olfactory neuroblastoma and neuroendocrine carcinoma may be difficult because a small proportion of SNUC may express neuroendocrine markers including NSE and chromogranin, SNUC, however, lacks S-100 and does not show evidence of glandular differentiation or rosettes. Cytological atypia and necrosis are also more extensive in SNUC. When neuroendocrine markers are observed in SNUC, they tend to be focal and no neurosecretory granules are evident ultrastructurally. ${ }^{5}$

Poorly differentiated squamous cell carcinomas usually show small areas of obvious squamous differentiation or keratinization and have a different cytokeratin pattern. Sinonasal undifferentiated carcinoma generally express simple epithelial type cytokeratins (CK 7, 8 and 19), while squamous carcinomas also express stratified epithelial keratins including CK 5, 6, 13 and 14. Negative staining for these latter cytokeratins would support a diagnosis of SNUC. ${ }^{6,7}$

Melanoma and lymphomas may also present as undifferentiated blue round cell tumors in the nasopharyngeal region. These tumors are easily discounted on the basis of immunohistochemistry. Melanomas stain positively for S-100, HMB 45 and melan A, while lymphomas are invariably positive for CD19 and CD20 as well as other B cell lineage markers. ${ }^{8}$

Sinonasal undifferentiated carcinoma commonly involves more anterior nasal cavity and ethmoids. Immunohistochemically, all SNUCs are positive for cytokeratin or EMA and many stain for both epithelial markers. Approximately one half of the tumors are positive for NSE, most lack S-100 immunoreactivity and all are negative for vimentin. ${ }^{3}$

The etiology of SNUC is unknown. There may be an association with cigarette smoking or a previous history of radiation therapy. ${ }^{2}$ In contrast to nasopharyngeal carcinoma, an association with Epstein-Barr virus infection has not been demonstrated. ${ }^{9}$

The most common symptoms are nasal obstruction, proptosis, periorbital swelling, diplopia, epistaxis, cranial nerve palsies and periorbital pain. The duration of symptoms can vary from a few weeks to 5 months. These tumors can involve multiple paranasal sinuses with marked destruction of sinus walls. Invasion of the orbit and the cranial cavity can occur, and they can spread to the nasopharynx. ${ }^{10}$ Most of these features are consistent with the findings in our case.

The radiologic feature of SNUC is a heterogeneous mass lesion causing bone destruction and invasion of adjacent structures, including the anterior cranial fossa, adjacent paranasal sinuses, and orbits. ${ }^{11}$ Bone destruction and involvement of adjacent structures were evident in our case.

Due to the small number of reported cases, the ideal treatment regimen has not been systematically evaluated. However, treatment generally involves surgical removal of the tumor. Surgical resection typically involves extensive craniofacial resection with maxillectomy, orbital exenteration and occasionally, neurosurgical involvement. Patients with SNUC have a high rate of both local regional recurrence and distant metastasis. Moreover, because of the complex anatomy of the head and neck area, complete removal of the tumor with wide margins is not always possible. The treatment of SNUC includes aggressive multimodality therapy, including surgical resection and adjuvant therapy (i.e. radiotherapy, chemotherapy). Chemotherapy agents used include carboplatin, cisplatin and etoposide. ${ }^{12,13}$

The prognosis associated with SNUC is poor, and death often occurs within a short span of time following the diagnosis. Loco-regional recurrences have been reported as high as $63 \%$ in a study by Kim et al. ${ }^{10}$ In the original report by Frierson et $\mathrm{al}^{1}{ }^{1}$ the median survival was only 4 months in patients of SNUC treated with radiotherapy alone. Improved survival (median 53.6 months) results for SNUC were reported by Deutsch et al ${ }^{14}$ by using aggressive multimodality approach. Lin et al in a retrospective review of 19 patients with SNUC reported an overall survival rate of $22 \%$ at 5 years, and the estimated 5-year distant metastasis free survival of $35 \%$. Local control was best in those patients treated nonsurgically, as was median survival, though this was not statistically significant. ${ }^{15}$

In a recent study by Chambers et al, a total of 318 cases of SNUC were identified between 1973 and 2010 in the 
United States from the surveillance, epidemiology, and end results (SEER) program of the National Cancer Institute. Five-year relative survival for those that received surgery, radiation and surgery combined with radiation was 38.7, 36.0 and 39.1\%, respectively. Median survival for surgery combined with radiation was 41.9 months. Median survival from 1973 to 1986 and 1986 to 2010 was 14.5 and 23.5 months, respectively; thereby confirming the survival benefit of surgery and radiation therapy and identifying a trend toward improvement in survival in recent decades. ${ }^{16}$

Despite advances in anterior skull base surgery and chemoradiation therapy, the cure-rate for SNUC remains dismally low. Recurrence rate is very high and many patients die of disease within months of diagnosis. Novel therapies that have been attempted include boron neutron capture therapy, autologous bone marrow transplant, and neoadjuvant selective intra-arterial cisplatin with concurrent radiation therapy, all of which showed limited success. ${ }^{17}$

\section{CONCLUSION}

It is important to recognize and differentiate this distinct entity from other paranasal sinus tumors because of its aggressive behavior, since early intervention may result in a better chance of survival and outcome.

\section{REFERENCES}

1. Frierson HF Jr, Mills SE, Fechner RE, Taxy JB, Levine PA. Sinonasal undifferentiated carcinoma: an aggressive neoplasm derived from schneiderian epithelium and distinct from olfactory neuroblastoma. Am J Surg Pathol 1986;10:771-779.

2. Frierson HF Jr. Sinonasal undifferentiated carcinoma. In: Barnes L, Eveson JW, Reichart P, Sidransky D, editors. World Health Organization classification of tumors. Lyon: IARC Press; 2005. p. 19.

3. Norleza AN, Gendeh BS. Challenges in the treatment of sinonasal undifferentiated carcinoma: a ray of hope. Med J Malaysia 2005 Aug;60(3):281-285.

4. Taxy JB, Bharani NK, Mills SE, Frierson HF Jr, Gould VE. The spectrum of olfactory neural tumors. A light microscopic, immunohistochemical and ultrastructural analysis. Am J Surg Pathol 1986;10:687-695.
5. Smith SR, Som P, Fahmy A, Lawson W, Sacks S, Brandwein M. A clinicopathological study of sinonasal neuroendocrine carcinoma and sinonasal undifferentiated carcinoma. Laryngoscope 2000;110:1617-1622.

6. Franchi A, Moroni M, Massi D, Paglierani M, Santucci M. Sinonasal undifferentiated carcinoma, nasopharyngeal type undifferentiated carcinoma, and keratinizing and nonkeratinizing squamous cell carcinoma express different cytokeratin patterns. Am J Surg Pathol 2002;26:1597-1604.

7. Jeng YM, Sung MT, Fang CL, Huang HY, Mao TL, Cheng W, et al. Sinonasal undifferentiated carcinoma and nasopharyngeal type undifferentiated carcinoma: two clinically, biologically, and histopathologically distinct entities. Am J Surg Pathol 2002;26:371-376.

8. Franquemont DW, Mills SE. Sinonasal malignant melanoma. A clinicopathologic and immunohistochemical study of 14 cases. Am J Clin Pathol 1991;96:689-697.

9. Cerilli LA, Holst VA, Brandwein MS, Stoler MH, Mills SE. Sinonasal undifferentiated carcinoma: immunohistochemical profile and lack of EBV association. Am J Surg Pathol 2001;25:156-163.

10. Kim B, Vongtama R, Juillard G. Sinonasal undifferentiated carcinoma: case series and literature review. Am J Otolaryngol 2004;25:162-166.

11. Phillips CD, Futterer SF, Lipper MH, Levine PA. Sinonasal undifferentiated carcinoma: CT and MR imaging of an uncommon neoplasm of the nasal cavity. Radiol 1997;202: 477-480.

12. Gorelick J, Ross D, Marentette L, Blaivas M. Sinonasal undifferentiated carcinoma: a case series and review of the literature. Neurosurg 2000;47(3):750-755.

13. Thompson L. Malignant neoplasms of the nasal cavity, paranasal sinuses, and nasopharynx. In: Thompson L, editor. Head and neck pathology. Philadelphia: Churchill Livingstone; 2006. p. 168-171.

14. Deutsch BD, Levine PA, Stewart FM, Frierson HF Jr, Cntrell RW. Sinonasal undifferentiated carcinoma: a ray of hope. Otolaryngol Head Neck Surg 1993;108:697-700.

15. Lin EM, Sparano A, Spalding A, et al. Sinonasal undifferentiated carcinoma : a 13-year experience at a single institution. Skull Base 2010;20:61-67.

16. Chambers KJ, Lehmann A, Remenschneider A, et al. Incidence and survival patterns of sinonasal undifferentiated carcinoma in the United States. J Neurol Surg B 2014;75-A066.

17. Xu CC, Dziegielewski PT, McGaw WT, Seikaly H. Sinonasal undifferentiated carcinoma (SNUC): the Alberta experience and literature review. J Otolaryngol-Head and Neck Surg 2013;42:2. 